\title{
Isolation and Biochemical Fingerprinting of Vancomycin-Resistant Enterococcus faecium From Meat, Chicken and Cheese
}

\author{
Malihe Talebi ${ }^{1}$; Javad Sadeghi ${ }^{2}$; Fateh Rahimi ${ }^{3,}$; Mohammad Reza Pourshafie ${ }^{4,}$ \\ ${ }^{1}$ Department of Microbiology, School of Medicine, Iran University of Medical Sciences, Tehran, IR Iran \\ ${ }^{2}$ Department of Microbiology, School of Medicine, Tehran University of Medical Sciences, Tehran, IR Iran \\ ${ }^{3}$ Department of Microbiology, Faculty of Science, University of Isfahan, Isfahan, IR Iran \\ ${ }^{4}$ Department of Microbiology, Faculty of Science, University of Isfahan, \\ ${ }^{*}$ Corresponding authors: Fateh Rahimi, Department of Microbiology, Faculty of Science, University of Isfahan, Isfahan, IR Iran. Tel: +98-3137932250, Fax: +98-3137932456, \\ E-mail: f.rahimi@sci.ui.ac.ir; Mohammad Reza Pourshafie, Department of Microbiology, Pasteur Institute of Iran, Tehran, IR Iran. Tel: +98-2166405534, Fax: +98-2166405535, \\ E-mail: pour@pasteur.ac.ir \\ Received: October 30, 2013; Revised: January 18, 2014; Accepted: February 12, 2014
}

\begin{abstract}
Background: Vancomycin-resistant enterococci (VRE) are important nosocomial pathogens and food chain has been considered as an assumed source for dissemination of VRE to human.

Objectives: The presence of VRE isolates from food samples and typing of these isolates with Phene plate, a biochemical fingerprinting method, were investigated.

Materials and Methods: Thirty samples of meat, chicken and cheese were analyzed for VRE during 2010. Antibiotic susceptibility tests and minimum inhibitory concentration (MIC) were also examined. VRE isolates were typed with the Phene plate system (PhPlate), a biochemical fingerprinting method.

Results: A total of 70 VRE isolates were obtained and identified as Enterococcus faecium by species-specific PCR. All the isolates carried vanA, while none of them harbored vanB. The VRE isolates included 35, 27, and 8 isolates from meat, chicken and cheese, respectively. Typing with the PhPlate revealed a diversity index of 0.78 for E. faecium, containing 10 common and four single types. The results of antibiotic susceptibility and MIC tests showed an increased resistance to ciprofloxacin, erythromycin, ampicillin and gentamicin, to which, 100\%, $100 \%, 100 \%$, and $95 \%$ of VRE isolates were resistant, respectively. Only $5 \%$ of the isolates were resistant to chloramphenicol and the MIC of the isolates for vancomycin and teicoplanin was $\geq 256 \mu \mathrm{g} / \mathrm{mL}$ and for gentamicin-resistant isolates it was $1024 \mu \mathrm{g} / \mathrm{mL}$. Conventional and molecular identification tests exhibited that all the isolates were E. faecium carrying vanA. None of the isolates harbored vanB.

Conclusions: The results showed that enterococci are common contaminants in food. Indeed, this study indicates a high prevalence of multidrug-resistant enterococci in food of animal origin in Iran. Isolating some persisting enterococcal isolates revealed that continuous surveillance of antimicrobial resistance in enterococci from food is essential.
\end{abstract}

Keywords: Fingerprinting; Food; Minimum Inhibitory Concentration; Multidrug Resistance; Vancomycin-Resistant Enterococci

\section{Background}

Enterococci are pervasive and predominant inhabitants of the gastrointestinal tract of humans and animals, found in soil, water and food (1). For many years, they were considered as normal flora and unharmful to man (2). However, over the last years, enterococci have emerged as major nosocomial pathogens, representing an increasingly important problem for public health (3). Indeed, these bacteria have a great ability to acquire resistance to some antimicrobial agents such as glycopeptides, in particular vancomycin, which are important for human therapy (4). Although enterococci are normally of relatively low virulence, they can transfer their antimicrobial resistance genes and virulence factors to other intestinal microflora and/or virulent bacteria, resulting in an increased pathogenicity (5).

The climbing incidence of antibiotic resistant Enterococcus spp. is the result of increased use of antibiotics in human health care system and animal growth promot- ers (6). The presence of large numbers of enterococci, in particular multidrug resistant ones, occurs commonly in vegetables, dairy and animal products (7). This dilemma, in part, is because of extensive usage of antimicrobial agents in modern farm industry (8). Due to limited therapeutic options for treating vancomycin-resistant enterococci (VRE), they are considered as a major cause of concern. VRE has been isolated from hospital sources, food animals, environment, and waste water. Previously, we investigated more about VRE from clinical samples, surface water and sewage treatment plants in Iran. The results of our prior studies showed isolation of a high number of VRE isolates from water and sewage $(9,10)$.

\section{Objectives}

In view of the lack of information about VRE isolates in food samples, we studied the occurrence of VRE in meat, chicken and cheese which were sold in local markets.

Copyright (C) 2015, Ahvaz Jundishapur University of Medical Sciences. This is an open-access article distributed under the terms of the Creative Commons Attribution-NonCommercial 4.0 International License (http://creativecommons.org/licenses/by-nc/4.0/) which permits copy and redistribute the material just in noncommercial usages, provided the original work is properly cited. 


\section{Materials and Methods}

\subsection{Sample Collection and Processing}

Thirty food samples, each 10 from chicken, meat and cheese were collected from Tehran local markets from April to September 2010. The samples were sealed in a plastic bag, labeled immediately and transported to a microbiology laboratory in a cold cycle. Ten grams of each sample were suspended in $90 \mathrm{~mL}$ of saline and then heavily vortexed. The mixture was filtered using $0.45 \mu$ filter membrane (Millipore, Sparks, MD, USA). The filters were then transferred to m-Enterococcus agar (Becton Dickinson and Co., Sparks, MD, USA) supplemented with $4 \mu \mathrm{g} /$ $\mathrm{mL}$ vancomycin and incubated for 48 hours at $37^{\circ} \mathrm{C}$.

\subsection{Identification of Isolates}

Colonies suspected to be Enterococcus were subjected to identification tests using the following characteristics: growth and hydrolysis of bile-esculin agar, growth in the presence of $6.5 \% \mathrm{NaCl}$, absence of catalase, presence of pyrrolidonyle arylamidase, $0.04 \%$ tellurite reduction, arabinose utilization, arginine dehydrolase activity, methyl-a-d-glucopyranoside acidification, and motility and pigmentation. Species identifications were confirmed by PCR using specific primers (11).

\subsection{Antibiotic Susceptibility Tests}

Minimum inhibitory concentration (MIC) for vancomycin, teicoplanin and gentamicin were determined using the E test (Biodisk AB, Solna, Sweden). Susceptibility tests to ampicillin $(10 \mu \mathrm{g})$, ciprofloxacin $(5 \mu \mathrm{g})$, gentamicin $(120 \mu \mathrm{g})$, erythromycin $(15 \mu \mathrm{g})$, tetracycline $(30 \mu \mathrm{g})$, chloramphenicol $(30 \mu \mathrm{g})$, linezolid $(30 \mu \mathrm{g})$, and quinupristin/ dalfopristin (Q-D) $(15 \mu \mathrm{g})$, (Mast Diagnostics Ltd., Bootle, Merseyside, UK) were performed by disc diffusion method according to the Clinical Laboratory Standard Institute (CLSI) guidelines (12). Enterococcus faecalis ATCC29212 was used as control.

\subsection{DNA Extraction and Polymerase Chain Reaction}

All the VRE isolates were investigated for vancomycin resistance genes. For DNA extraction, one isolated colony from each plate was transferred into $200 \mu \mathrm{L}$ distilled water and boiled at $100^{\circ} \mathrm{C}$ for 15 minutes. The mixture was centrifuged and $10 \mu \mathrm{L}$ of the supernatant was used as the DNA template in the PCR mix. Identification of van genotypes (vanA, vanB) for each isolate of VRE was performed by a separate PCR with specific primers as follows: vanA, 5'-CATGAATAGAATAAAAGTTGCAATA-3', 5'-CCCCTTTAACGCTAATACGATCAA-3'; vanB, 5'-GTGACAAACCGGAGGCGAGGA-3', 5'-CCGCCATCCTCCTGCAAAAAA-3'. The PCR assay was performed in a total volume of $25 \mu \mathrm{L}$ containing $10 \mathrm{mM}$ Tris- $\mathrm{HCl}(\mathrm{pH}=8.3)$, $1.5 \mathrm{mM} \mathrm{MgCl}_{2}, 0.2 \mathrm{mM}$ each dNTPs, $0.5 \mathrm{U}$ TaqDNA poly- merase (HT Biotechnology, Cambridge, UK), and each primer (40 pmol). The PCR cycle was as follows; initial denaturation at $94^{\circ} \mathrm{C}$ for five minutes, 30 cycles of denaturation at $94^{\circ} \mathrm{C}$ for one minute, annealing at $54^{\circ} \mathrm{C}$ for one minute and extension at $72^{\circ} \mathrm{C}$ for one minute, with a final extension at $72^{\circ} \mathrm{C}$ for 10 minutes (13). The PCR was performed with an Eppendorf Mastercycler (Eppendorf, Hauppauge, NY).

\subsection{Typing of Vancomycin-Resistant Enterococci Isolates}

All the isolated VRE were typed using a high-resolution biochemical fingerprinting method, Phene plate (PhPlate) system, which is specifically developed for typing of enterococci strains (The PhPlate AB, Stockholm, Sweden). Microplates with 11 carbohydrate substrates were specifically chosen to differentiate between strains of enterococci. Preparation and inoculation of the plates were according to the manufacturer instruction. Briefly, a loopful of a fresh bacterial culture was inoculated in PhPlate growth media containing $0.2 \%(\mathrm{w} / \mathrm{v})$ protease peptone, $0.05 \%(\mathrm{w} / \mathrm{v})$ yeast extract, $0.5 \%(\mathrm{w} / \mathrm{v}) \mathrm{NaCl}$ and $0.011 \%(\mathrm{w} / \mathrm{v})$ bromothymol blue. The plates were then incubated at $37^{\circ} \mathrm{C}$ and images of the plates were scanned after 16, 24 and 48 hours using an HP Scanjet 4890 scanner. After the final scan, the PhPlate software (PhPWin 4.2) was used to create the absorbance data (biochemical fingerprint) from the scanned images. The correlation coefficient using a pair-wise comparison of the biochemical fingerprints and clustered was determined according to the unweighted pair group method (UPGMA) with arithmetic averages. The mean similarity between the compared isolated minus 2SD was taken as the ID-level of the system. Isolates showing similarity to each other above this level were considered as identical (Common Biochemical Phenotypes: C-BPT) (13).

\section{Results}

\subsection{Vancomycin resistant Enterococci Detection and Identification}

According to the biochemical tests, a total of 102 isolates belonged to enterococci, 48, 40 and 14 of which were from meat, chicken and cheese, respectively. Antibiotic susceptibility test revealed that 35, 27 and 8 isolates obtained from meat, chicken and cheese, respectively, were vancomycin-resistant. Conventional and molecular identification tests exhibited that all the isolates were E. faecium carrying vanA. None of the isolates harbored vanB.

\subsection{Antibiotic Resistance}

Using the method of CLSI, all the isolates were tested for their resistance against antimicrobial agents. All the 
VRE isolates were susceptible to linezolid, quinupristindalfopristin and tetracycline. However, they showed different degrees of resistance to other antibiotics. All the VRE isolates were also resistant to ciprofloxacin, erythromycin and ampicillin (100\%). Almost the same antibiotic resistance was observed for gentamicin (95\%), but only $5 \%$ of the isolates were resistant to chloramphenicol. The MIC of the isolates for vancomycin and teicoplanin was $\geq 256 \mu \mathrm{g} / \mathrm{mL}$ and for gentamicin-resistant isolates it was $1024 \mu \mathrm{g} / \mathrm{mL}$.

\subsection{Typing}

The results of typing with PhPlate system showed a diversity of $\mathrm{Di}=0.78$ for the E. faecium population. A total of 14 types with 10 common types (C-BPT) constituting 66 isolates and four single types (S-BPT) were seen (Figure 1). Each common type comprised 2 - 31 strains. The VRE from the meat samples showed 11 types comprising 35 isolates, while the chicken isolates contained only four types. Some of the isolates collected from different food samples on different sampling occasions were found to have the same C-BPT (Table 1).

Table 1. Number of Vancomycin resistant Enterococci Strains in Each Phene Plate Type and Their Sample Types and Number of Sampling ${ }^{\text {a }}$

\begin{tabular}{|c|c|c|c|}
\hline BPT & $\begin{array}{l}\text { Number of } \\
\text { VRE Isolates }\end{array}$ & $\begin{array}{l}\text { Number of } \\
\text { Sampling }\end{array}$ & Sample Type \\
\hline CT1 & 3 & 1,2 & Meat \\
\hline CT2 & 2 & 1,2 & Meat \\
\hline CT3 & 4 & 3,5 & Meat \\
\hline CT4 & 2 & 4,6 & Meat, chicken \\
\hline CT5 & 3 & 1,3 & Meat \\
\hline CT6 & 4 & 1,6 & Meat \\
\hline CT7 & 2 & 2,4 & Meat \\
\hline CT8 & 2 & 1,7 & Meat, chicken \\
\hline CT9 & 31 & $1,2,3,4,6,7,8,9,10$ & $\begin{array}{l}\text { Meat, chicken, } \\
\text { cheese }\end{array}$ \\
\hline CT10 & 13 & $2,3,4,6,7,8$ & Chicken, cheese \\
\hline ST1 & 1 & 3 & Meat \\
\hline ST2 & 1 & 4 & Meat \\
\hline ST3 & 1 & 10 & Cheese \\
\hline ST4 & 1 & 7 & Cheese \\
\hline
\end{tabular}

a Abbreviations: BPT, biochemical phenotype; CT: common type; and ST: single type.

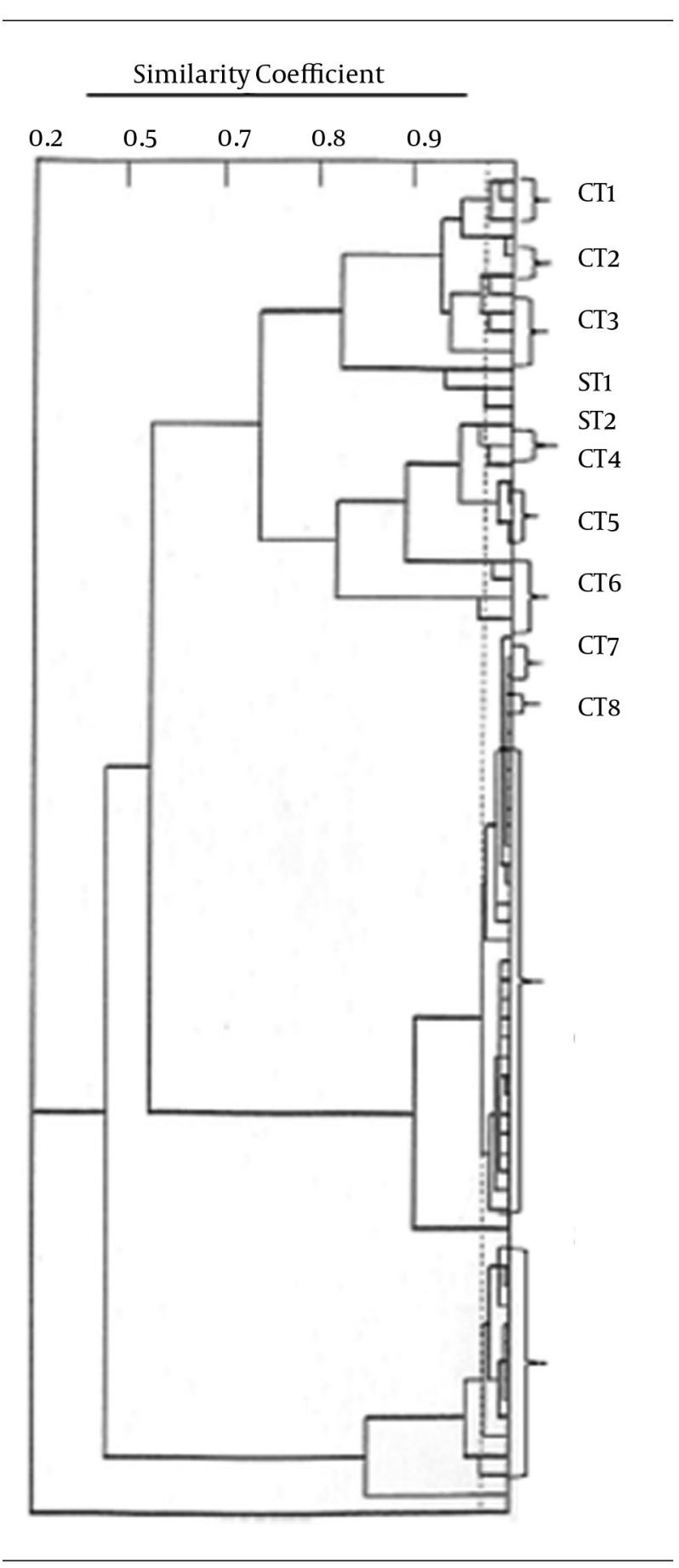

Figure 1. A Unweighted Pair Group Method Dendrogram Showing 70 Enterococcus faecium Strains Isolated From Different Samples

\section{Discussion}

A high rate of vanA containing Enterococcus isolates was detected in food samples of chicken (9/10) and meat (10/10) in this study. Isolation of vanA-containing enterococci strains in poultry and fresh slaughtered chicken samples has been reported in Germany (14). Furthermore, high-level glycopeptide-resistant VanA-type strains 
Talebi M et al.

from supermarket-purchased chicken were detected in England (15). On the other hand, absence of VRE from meats was reported in studies performed in the US $(16$, 17) which reflected the absence of VRE isolation in animal food product. Elimination of VRE, in part, may have been resulted during the food processing. On the contrary, in European countries, VRE have been frequently isolated from meat products, which might be due to the usage of glycopeptides avoparcin antibiotic in food animal production environments before banning this antibiotic $(18,19)$. In this study, we obtained similar results to the European countries which may indicate the presence of similar diets in Iran.

Our results showed the presence of enterococci strains in $50 \%$ of cheese samples. In contrast, in a study by Giraffa and Sisto, there was no evidence of VRE in dairy products (20). However, some years later, Giraffa and colleagues in Italy found that $50 \%$ of the cheeses examined were contaminated by VRE (7). E. faecium was the only species isolated in all the samples in our study, while in most of the studies E. faecium and E. faecalis were simultaneously isolated from food samples. Klein et al. reported a total of 34 VRE strains isolated from raw minced beef and pork and $38 \%$ of VRE isolates were identified as E. faecium, 35\% were E. faecalis, and the remaining isolates were from the E. faecium group (21). Moreover, similar results were detected in the UK in fresh and frozen chicken, 58\% and 40\% of which were E. faecium and E. faecalis, respectively (15).

Antibiotic susceptibility tests showed that in the present study, the VRE isolates were resistant to at least four antibiotics including gentamicin, ciprofloxacin, erythromycin and ampicillin. This has been confirmed by other studies which have found the prevalence of antibiotic-resistant enterococci in farm animals and their meat to be higher than $60 \%(22,23)$. These studies showed that extensive agricultural use of glycopeptides or other antibiotics has created animal reservoir of resistant enterococcal species to antibiotics which has complicated the control of infections caused by enterococci. Here we determined that resistance to gentamicin was very high among the animal products (95\%). Using the synergism between aminoglycosides and B-lactams or glycopeptides eliminated owing to high-level aminoglycoside resistance, which is of vast clinical importance (24). Peters and colleagues have reported a very low gentamicin resistance in food from animal origin in Germany (25). The difference in the reported antibiotic resistance among animal products could be due to geographical differences and the policy as well as production practices performed in these countries.

All of our isolates were susceptible to tetracycline, in comparison with the results from Peters and colleagues who found a high rate of tetracycline resistance in their strains (25). The reason for the absence of resistance to tetracycline in this study may be due to the fact that tetracycline is not used as a therapeutic antimicrobial in veterinary medicine in Iran. In consistent with another study, here we reported that the prevalence of chloramphenicol resistance was very low among E. faecium strains (26).We found no resistant isolate to oxazolidinone, linezolid and Q-D in this study (25). Although surveillance of enterococci from food sources for resistance to linezolid has not been reported extensively (26), there are some resistance reports to Q-D in the USA, given the use of the analogue virginiamycin since $1974(27,28)$.

In PhPlate analysis, we found that some of the C-BPT were found on different sampling occasions and food samples (i.e. C8, C9), indicating a high prevalence of certain E. faecium C-BPT in the food. In addition, in another study, typing with PhPlate exhibited a high diversity in a large number of enterococci isolated from different sources including food samples (29). On the other hand, genotyping with Pulse-Field Gel Electrophoresis profile (PFGE) and Random Amplification of Polymorphic DNA (RAPD) PCR revealed a marked heterogeneity in food isolates $(30,31)$. These results also showed that isolates with identical BPT pattern were found in different sampling occasions in the same food sample (i.e. isolates in C10), suggesting that some of the strains persisted for a period of time.

In conclusion, the high prevalence of multidrug resistance among enterococci isolated from food is a serious threat to public health. To the best of our knowledge, there is no use of antibiotic other than human use, in animal feeding in Iran. The data presented here, on the other hand, suggested the presence of antibiotic pressure in food animals. The level of antibiotics in animal product, in turn, may be due to treatment regimens used for infections in animals. Controlled use of antibiotics in animal husbandry is highly suggestive in Iran.

\section{Authors' Contributions}

Study concept and design: Fateh Rahimi, Mohammad Reza Pourshafie. Acquisition of data: Malihe Talebi, Javad Sadeghi, Fateh Rahimi. Analysis and interpretation of data: Malihe Talebi, Fateh Rahimi. Drafting of the manuscript: Malihe Talebi, Javad Sadeghi. Critical revision of the manuscript for important intellectual content: Mohammad Reza Pourshafie. Administrative, technical, and material support: Fateh Rahimi, Mohammad Reza Pourshafie. Study supervision: Mohammad Reza Pourshafie.

\section{References}

1. Pedonese F, Innocenti E, Nuvoloni R, Sartini L, D'Ascenzi C, Cerri $D$, et al. Occurrence of vancomycin-resistant enterococci in foods of animal origin purchased in Tuscany. Vet Res Commun. 2005;29 Suppl 2:347-9.

2. Wierzchowska WC, Zadernowska A, Nalepa B, Laniewska TL. Occurrence and antibiotic resistance of enterococci in ready-to-eat food of animal origin. Afr J Microbiol Res. 2012;6(39):6773-80.

3. Sood S, Malhotra M, Das BK, Kapil A. Enterococcal infections \& antimicrobial resistance. Indian J Med Res. 2008;128(2):111-21.

4. Arias CA, Murray BE. The rise of the Enterococcus: beyond vancomycin resistance. Nat Rev Microbiol. 2012;10(4):266-78.

5. McGowan-Spicer LL, Fedorka-Cray PJ, Frye JG, Meinersmann RJ, Barrett JB, Jackson CR. Antimicrobial resistance and virulence 
of Enterococcus faecalis isolated from retail food. J Food Prot. 2008;71(4):760-9.

6. Aarestrup FM. Occurrence, selection and spread of resistance to antimicrobial agents used for growth promotion for food animals in Denmark. APMIS Suppl. 2000;101:1-48.

7. Giraffa G, Olivari AM, Neviani E. Isolation of vancomycin-resistant Enterococcus faecium from Italian cheeses. Food Microbiol. 2000;17(6):671-7.

8. Heuer OE, Hammerum AM, Collignon P, Wegener HC. Human health hazard from antimicrobial-resistant enterococci in animals and food. Clin Infect Dis. 2006;43(7):911-6.

9. Pourshafie MR, Talebi M, Saifi M, Katouli M, Eshraghi S, Kuhn I, et al. Clonal heterogeneity of clinical isolates of vancomycinresistant Enterococcus faecium with unique vanS. Trop Med Int Health. 2008;13(5):722-7.

10. Talebi M, Rahimi F, Katouli M, Kühn I, Möllby R, Eshraghi S, et al. Prevalence and Antimicrobial Resistance of Enterococcal Species in Sewage Treatment Plants in Iran. Water Air Soil Pollut. 2007;185:111-9.

11. Jackson CR, Fedorka-Cray PJ, Barrett JB. Use of a genus- and species-specific multiplex PCR for identification of enterococci. J Clin Microbiol. 2004;42(8):3558-65.

12. Clinical and Laboratory Standards Institute . Performance standards for antimicrobial disc diffusion tests. Approved standards. 2012. Available from: http://antimicrobianos.com.ar/ATB/wpcontent/uploads/2012/11/01-CLSI-M02-A11-2012.pdf.

13. Talebi M, Rahimi F, Katouli M, Mollby R, Pourshafie MR. Epidemiological link between wastewater and human vancomycin-resistant Enterococcus faecium isolates. Curr Microbiol. 2008;56(5):468-73.

14. KlareI, Heier H, Claus H, Bohme G, Marin S, Seltmann G, et al.Enterococcus faecium strains with vanA-mediated high-level glycopeptide resistance isolated from animal foodstuffs and fecal samples of humans in the community. Microb Drug Resist. 1995;1(3):265-72.

15. Kirk M, Hill RL, Casewell MW, Beighton D. Isolation of vancomycin-resistant enterococci from supermarket poultry. Adv Exp Med Biol. 1997; 418:289-91.

16. Thal LA, Chow JW, Mahayni R, Bonilla H, Perri MB, Donabedian SA, et al. Characterization of antimicrobial resistance in enterococci of animal origin. Antimicrob Agents Chemother.1995;39(9):2112-5.

17. Coque TM, Tomayko JF, Ricke SC, Okhyusen PC, Murray BE. Vancomycin-resistant enterococci from nosocomial, community, and animal sources in the United States. Antimicrob Agents Chemother. 1996;40(11):2605-9.

18. Lemcke R, Bulte M. Occurrence of the vancomycin-resistant genes vanA, vanB, vanCl, vanC2 and vanC3 in Enterococcus strains isolated from poultry and pork. Int J Food Microbiol. 2000;60(2-3):185-94.

19. Quednau M, Ahrne S, Petersson AC, Molin G. Antibiotic-resistant strains of Enterococcus isolated from Swedish and Danish retailed chicken and pork. J Appl Microbiol. 1998;84(6):1163-70.

20. Giraffa G, Sisto F. Susceptibility to vancomycin of enterococci isolated from dairy products. Lett Appl Microbiol.1997;25(5):335-8.

21. Klein G, Pack A, Reuter G. Antibiotic resistance patterns of enterococci and occurrence of vancomycin-resistant enterococci in raw minced beef and pork in Germany. Appl Environ Microbiol. 1998;64(5):1825-30.

22. Giraffa G. Enterococci from foods. FEMS Microbiol Rev. 2002;26(2):163-71.

23. Teuber M, Meile L, Schwarz F. Acquired antibiotic resistance in lactic acid bacteria from food. Antonie Van Leeuwenhoek. 1999;76(1-4):115-37.

24. Murray BE. The life and times of the Enterococcus. Clin Microbio Rev.1990;3(1):46-65.

25. Peters J, Mac K, Wichmann-Schauer H, Klein G, Ellerbroek L. Species distribution and antibiotic resistance patterns of enterococci isolated from food of animal origin in Germany. Int $J$ Food Microbiol. 2003;88(2-3):311-4.

26. Hayes JR, English LL, Carter PJ, Proescholdt T, Lee KY, Wagner $\mathrm{DD}$, et al. Prevalence and antimicrobial resistance of enterococcus species isolated from retail meats. Appl Environ Microbiol. 2003;69(12):7153-60.

27. Hayes JR, McIntosh AC, Qaiyumi S, Johnson JA, English LL, Carr LE, et al. High-frequency recovery of quinupristin-dalfopristin-resistant Enterococcus faecium isolates from the poultry production environment. J Clin Microbiol. 2001;39(6):2298-9.

28. Welton LA, Thal LA, Perri MB, Donabedian S, McMahon J, Chow JW, et al. Antimicrobial resistance in enterococci isolated from Turkey flocks fed virginiamycin. Antimicrob Agents Chemother. 1998;42(3):705-8.

29. Kuhn I, Iversen A, Mollby R. The PhenePlate system for studies of the diversity of enterococcal populations from the food chain and the environment. Int J Food Microbiol. 2003;88(2-3):189-96.

30. Gomes BC, Esteves CT, Palazzo IC, Darini AL, Felis GE, Sechi LA, et al. Prevalence and characterization of Enterococcus spp. isolated from Brazilian foods. Food Microbiol. 2008;25(5):668-75.

31. Vignaroli C, Zandri G, Aquilanti L, Pasquaroli S, Biavasco F. Multidrug-resistant enterococci in animal meat and faeces and cotransfer of resistance from an Enterococcus durans to a human Enterococcus faecium. Curr Microbiol. 2011;62(5):1438-47. 